Sains Malaysiana 49(9)(2020): 2043-2051

http://dx.doi.org/10.17576/jsm-2020-4909-02

\title{
Temporal Discrete Z-Number and Its Application in Assessing EEG Signal Data of Epileptic Seizure
}

\author{
(Nombor-Z Diskret Temporal dan Aplikasinya dalam Menilai Data Signal EEG Sawan Epilepsi)
}

\author{
MUjahid ABDUllahi, TAHIR AHMAD* \& VinOD RAMACHANDRAN
}

\begin{abstract}
Analysis and modeling of a complex physical system, particularly EEG signals involved vague and uncertain information. The approach introduced by Kosanovic using temporal fuzzy set to model a complex system particularly the EEG signal does not address the problem of uncertainty for the time of occurrence. In this paper, an ordered discrete Z-number is used to construct temporal discrete Z-number to assess EEG signal data of an epileptic seizure for the first time. The proposed temporal discrete Z-number is able to accommodate the problem of uncertainty with regards to the time of occurrence for a given seizure by using and modifying the method for measuring the uncertainty of Z-number.
\end{abstract}

Keywords: Discrete Z-number; dynamic system; fuzzy set; uncertainty; Z-number

ABSTRAK

Pemodelan dan analisis sesuatu sistem yang kompleks, khususnya tentang kesamaran dan kebolehpercayaan melibatkan maklumat isyarat EEG itu sendiri. Pendekatan yang diperkenalkan oleh Kosanovic menggunakan set kabur temporal bagi memodelkan sesuatu sistem yang kompleks tidak menangani masalah ketidakpastian masa kejadian akan maklumat yang tercerap. Dalam makalah ini, nombor-Z diskret tertib digunakan bagi membina nombor-Z diskret temporal untuk menganalisis isyarat EEG yang tercerap ketika serangan sawan, diperkenalkan buat julung kalinya. Nombor-Z diskret temporal mampu menangani masalah ketidakpastian berhubung dengan pemasalahan masa kejadian bagi sesuatu serangan sawan dengan menggunakan pengubahsuaian yang dibuat terhadap kaedah mengukur ketidakpastian bagi nombor-Z.

Kata kunci: Ketidakpastian; nombor-Z; nombor-Z diskret; set kabur; sistem dinamik

\section{INTRODUCTION}

Electroencephalography (EEG) is a method used in clinical studies for analyzing the electrical activity of the brain. It is among the available methods used to identify abnormalities of the brain (Fauziah 2008). In particular, EEG plays significant diagnostic roles in epilepsy and provides supporting evidence of seizure disorder (Sharmila \& Geethanjali 2019). Hence, the analysis of EEG signals recorded during a seizure is crucial.

A universal method for signal and system analysis using fuzzy set was introduced in Kosanovic (1996), by utilizing temporal fuzzy set (TFS) to model the dynamic motion of EEG signals. Fuzzy information space, which is based on the membership functions of its temporal fuzzy set, is then constructed by Kosanovic. However, the proposed concept (TFS) does not address the uncertainty time of occurrence. The concept of Z-number was introduced by Zadeh (2011) to accommodate fuzziness and uncertainty of information concurrently.

A method for measuring uncertainty of fuzzy sets is developed (Kang et al. 2018). Kang modified Kosko's work (Kosko 1990) which is a distance-based fuzziness measure of the fuzzy set. Moreover, using the proposed approach, a method for measuring uncertainty for Z-numbers (discrete and continuous) is constructed where Z-number is viewed as a unique fuzzy structure with inherent uncertainty.

The motivation of this study is to propose a more general method for assessing EEG signals based on Z-number and to address the issue of uncertainty with respect to the time of occurrence. In this paper, the concept of temporal discrete Z-number (TDZ) is introduced for the first time to model complex system, particularly to serve as a tool for assessing EEG signal data of epileptic seizures, and it has the ability to model the uncertainty with respect to the time of occurrence.

\section{PRELIMINARIES}

Definition 1 (Casasnovas \& Riera 2006) A fuzzy number $A$ of the real line $R$ with membership function $\mu_{A}: \mathbb{R} \rightarrow[0,1]$ is a discrete fuzzy number if its support is finite, i.e. there exist $\left\{x_{1}, \ldots, x_{n}\right\} \in R$ with $x_{1}<x_{2}<\ldots<$ 
$x_{\mathrm{n}}$, such that $\operatorname{supp}(\mathrm{A})=\left\{x_{1}, \ldots, x_{n}\right\}$ and there exist natural numbers $s$, $t$ with $1 \leq s \leq t \leq$ satisfying the following conditions:

1. $\mu_{A}\left(x_{i}\right)=1$ for any natural number $i$ with $s \leq i \leq t$

2. $\mu_{A}\left(x_{i}\right) \leq \mu_{A}\left(x_{j}\right)$ for each natural number $i, j$ with 1 $\leq i \leq j \leq s$

3. $\mu_{A}\left(x_{i}\right) \geq \mu_{A}\left(x_{j}\right)$ for each natural number $i, j$ with $t$ $\leq i \leq j \leq n$

Definition 2 (Mendel \& John 2002) A (general) type2 fuzzy set $E$, is characterized by a type- 2 membership function $\mu_{E}(x, u)$, where $x \in X$ and $J_{x} \subseteq[0,1]$ :

$$
\begin{aligned}
E= & \left\{\left((x, u), \mu_{E}(x, u)\right) \mid \forall x \in X, \forall u \in J_{x} \subseteq[0,1]\right\}, \\
& 0 \leq \mu_{E}(x, u) \leq 1
\end{aligned}
$$

Definition 2 (Aliev et al. 2015) A discrete Z-number is an ordered pair $Z=A B$ where $A$ is a discrete fuzzy number playing a role as a fuzzy constraint on values that a random variable $X$ may take:

\section{$X$ is $A$}

and $B$ is a discrete fuzzy number with a membership function $\mu_{B}:\left\{b_{1}, \ldots, b_{n}\right\} \rightarrow[0,1],\left\{b_{1}, \ldots, b_{n}\right\} \subseteq[0,1]$, playing the role of a fuzzy constraint on the probability measure of $A: P(A)$ is $B$.

Definition 3 (Abdullahi et al. 2020) Let $Z_{D}$ be a discrete Z-number and let $\bar{Z}_{D}$ be a set of discrete Z-numbers, i.e. $Z_{D} \in \bar{Z}_{D}$. The pair $\left(Z_{D}, \prec\right)$ is called an ordered discrete $Z$-number, if there exists a relation $\prec$, such that $\left(\bar{Z}_{D}, \prec\right)$ is totally ordered.

Definition 4 (Kosanovic 1996) Let $\left(R, d_{R}\right)$ and $\left(M, d_{M}\right)$ be metric spaces, where $(M, \prec)$ is linearly ordered set with the minimal element $\alpha_{0} \in M$ and linear order $\prec$. Let $R^{M}$ be a set of all functions $h: M \rightarrow R$. A dynamic system is a collection $g(\alpha, \rho, \beta)$ of the transformation of the space $R \times M,(\rho, \beta) \in R \times M$ into $R^{M}, g(., \rho, \beta) \in R^{M}$, satisfying the following conditions:
a) $g\left(\alpha, \rho, \alpha_{0}\right)=\rho$
b) $g\left(\alpha, \rho, \alpha_{0}\right)$ is defined for all $\alpha_{0}$,
c) $g\left(\alpha, \rho, \alpha_{0}\right)$ is unique, i.e.g $\left[\alpha_{2}, g\left(\alpha_{1}, \rho, \alpha_{0}\right), \alpha_{1}\right]$ $=g\left(\alpha_{2}, \rho, \alpha_{0}\right)$ for all $\alpha_{1}, \alpha_{2}$
d) $g(\alpha, \rho, \beta)$ is continuous in all arguments.

Point $(\rho, \beta) \in R \times M$ is called an initial point. The function $g(., \rho, \beta)$, for a fixed $(\rho, \beta)$ is called a motion. The set of points $\mathrm{X}=\{g(\alpha, \rho, \beta): \alpha \in M\}$, for a fixed $(\rho, \beta)$, is called a trajectory of this motion. The set of ordered pairs $X \alpha=\{(v, \alpha): v=g(\alpha, \rho, \beta), \alpha \in M\}$ for a fixed $(\rho, \beta)$, is called an augmented trajectory of the motion.

Time is one of the significant features in some real-world problems, which is monotonic and it is a fundamental aspect for modeling dynamic information and systems, such as in the realms of decision making, traffic, weather, medicine, economics, and signal analysis. Time has two forms of representation which are; time interval and time instance. Time interval represents the range between two points in time while time instance represents a point in time. Most of the temporal models are based on either one of these forms (Rangasamy 2009). It was mentioned in Nagypál and Motik (2003) and Rangasamy (2009) that temporal information is not crisp, but it is uncertain and vague.

\section{TEMPORAL DISCRETE Z-NUMBER}

Basically, a temporal discrete Z-number is a discrete $Z$-number created from a universal set whose elements are ordered in time. Therefore, if the universe is an ordered set, it may contain an ordered discrete Z-number $Z^{\prec}$ with corresponding membership function $\mu_{Z^{<}}(\cdot)$. The elements in the universe $S$ are ordered via linear ordering relation $\prec$, where $\mu_{Z^{<}}(\cdot)=\left(\mu_{A}(\cdot), \mu_{B}(\cdot)\right)$, such that $\mu_{A}(\cdot)$ and $\mu_{B}(\cdot)$ are the membership functions of components of temporal discrete $\mathrm{Z}$-number.

A family of temporal discrete $Z$-number is constructed based on the idea of temporal fuzzy set introduced by Kosanovic (1996), to model dynamic motions of complex physical systems such as EEG. Unlike Kosanovic's work, this proposed structure addresses the issue of uncertainty time of occurrence. There are two approaches to the construction of temporal discrete Z-number. The first method is based on the inverse principle, which is explained as follows;

Let analyze a physical system determined by an ordinary differential equation

$$
\frac{d s}{d t}=F(s, t)
$$

where $t \in T=\left[t_{0}, \infty\right), s \in F^{p}$ and $G(\because)$ is a real-valued vector function that is Lipschitz continuous on a rectangle $\Gamma \subset T \times F^{p}$. The unique solution of equation (1), given initial conditions $\left(t_{0}, \mathrm{~s}_{0}\right)$ is written in vector form as

$$
s_{t}=S(t)
$$

such that $S(\cdot)$ is the state space trajectory for a particular initial condition $\left(t_{0}, \mathrm{~s}_{0}\right)$. By considering an observation interval $T_{0}=\left(t_{0}, t_{1}\right)$, and utilizing the state space trajectory $S($.$) , a universe of objects can be generated S^{*}=S\left(T_{0}\right)$ with its elements $S_{t}$ in temporal form. Given an arbitrary discrete $Z$-number $Z_{1} \in S^{*}$, the vector function creates a 
discrete $Z$-number $Z_{2}$ in $T_{0}$ through the inverse principle. Therefore, the membership function of $Z_{2}$ is defined as

$$
\mu_{Z_{1}}(t)=\mu_{Z_{1}}(r), r \in\left(S^{*}, \prec\right)
$$

such that $\mu_{Z_{1}}(t)=\left(\mu_{A_{1}}(t), \mu_{B_{1}}(t)\right)$ and $\mu_{Z_{1}}(r)=\left(\mu_{A_{1}}\right.$ $\left.(r), \mu_{B_{1}}(r)\right) \forall t \in T_{0}$ belong to the inverse image of $r$, that is $\forall t \in S^{-1}(r)$. The symbol $\prec$ means that the universe $S^{*}$ is ordered in time. The developed discrete Z-number $Z$, created from the dynamic trajectory $S^{*}$ is a temporal discrete $\mathrm{Z}$-number since the observation interval $T_{0}$ is ordered in time.

The temporal discrete Z-number generated from a discrete $Z$-number $Z_{1}$ in $\left(S^{*}, \prec\right)$ can be denoted as $Z^{\prec}$ and $Z_{1}$ is simply denoted as $Z$. The membership functions $\mu_{Z^{<}}(t)$ maps the observation interval $T_{0}$ into $[0,1]$. Therefore, the effect of discrete Z-number in the state space trajectory $S^{*}$ with respect to the general dynamic behavior can be measured at each time instant $t \in T_{0}$ with the membership value

$$
\mu_{Z^{<}}(t)=\mu_{Z}\left(s_{t}\right)
$$

Obviously, membership functions of temporal discrete Z-numbers are functions of time now. In reality, the state space trajectory may not be available. Therefore, let examine a set of feature samples $S$, obtained from different signals, e.g. EEG signal during an epileptic seizure, over a time interval $T$. Samples of $S$ exist in a feature space $F$ that describes the process. The samples make up a feature space trajectory that is expressed in terms of a vector-valued function $S: T \rightarrow F$, is, $S=S(T)$. Now, let $Z$ be a discrete $Z$-number that represents a property, e.g. stages of seizure, and $\mu_{z}(s)$ be the degree of seizure for a sample $s \in S$. Since the elements of $S$ are ordered in time, a discrete Z-number $Z^{\prec}$ in $T$ can be created as

$$
\mu_{Z^{<}}(t)=\mu_{Z}\left(s_{t}\right), s_{t} \in(S, \prec)
$$

for all $t \in T$.

The temporal discrete $\mathrm{Z}$-number is constructed to describe the regions of attraction in a space where the trajectory lies, which in general, is the feature space $F$, and the feature space trajectory $(S, \prec)$ such as the potential difference, frequency, and amplitude. Any of these can be considered as the feature space.

The temporal discrete Z-number constructed in (2) is not always applicable since it does not allow the membership values of a temporal discrete Z-number to depend completely on the time variable. i.e. the inverse principle of creating discrete $\mathrm{Z}$-numbers via functional maps forces $\mu_{Z^{<}}\left(t_{1}\right)=\mu_{Z}\left(s_{t_{2}}\right)$, whenever the corresponding points $Z_{1} Z_{2} \in F$ are equal. This may not be acceptable in all cases, e.g. time-varying dynamic processes. In such cases there may be points in feature space that are revisited at different time instants, e.g. there exist $M \subset T, e(M)>1$, and there exists $S \in F$ such that $s=g(t), t \in M$, where $e(\cdot)$ returns the cardinality and $g$ is the dynamic motion.

Therefore, a temporal discrete Z-number can be derived from the basic principle as follows; Let consider a physical process represented by its motion, $\left(F, d_{F}\right)$ is a feature space that characterizes the dynamic process. It is a finite-dimensional real vector space $F=\mathbb{R}^{p}$ with $p \in \mathbb{N}$ and $\left(T, d_{T}\right)$ represents time such that $\mathrm{T}=\left[t_{0}, \infty\right]$. The pair is linearly ordered set with the minimal element. Both $\left(F, d_{F}\right)$ and $\left(T, d_{T}\right)$ are metric spaces with the standard Euclidean distance function.

Using Definition 4 of a dynamic system and letting $s=Z$, such that $Z=(A, B)$ is an ordered discrete Z-number and by fixing $\left(Z_{0}, T_{0}\right) \in \mathrm{Q} \times T$ then the corresponding motion $g: T \rightarrow F$ defined as $g(t)=f(t$, $\left.Z_{0}, T_{0}\right) \forall t \in T$ where $f\left(t, Z_{0}, \tau\right)$ is the dynamic system that generates the physical process. The set of points $S$ $=\{g(t): t \in T\}$ is a trajectory of this motion and the set $S_{t}=\{(Z, t): Z=g(t), t \in T\}$ is the augmented trajectory of this motion. The set is an ordered set since $(Z, t) \in S$ is linearly ordered by applying the relation $\leq$ on $T$. In other words, the EEG data can be analyzed piece by piece or by partitioning it.

Definition 5 Let $\left(F, d_{F}\right)$ and $\left(T, d_{T}\right)$ be metric spaces, where $(T, \prec)$ such as the potential difference, frequency, and ampli is a linearly ordered set with a minimal element $t_{0} \in T$. Let $S_{t} \subset F \times T$ be an augmented trajectory of a dynamic motion $g \in F^{T}$ defined for all $t \in T$. The relation $\prec^{\prime}$ on $S_{t} \times S_{t}$, generated by $g(\cdot)$, is called a temporal ordering on $S_{t}$, and is defined as $\forall\left(Z_{1}, t_{1}\right),\left(Z_{2}, t_{2}\right) \in$ $S_{t}\left(Z_{1}, t_{1}\right) \prec^{\prime}\left(Z_{2}, t_{2}\right) \Leftrightarrow t_{1} \prec \mathrm{t}$, where $Z_{1}$ and $Z_{2}$ are ordered discrete Z-numbers. For any set $K_{\mathrm{t}} \subseteq S_{\mathrm{t}}$, a pair $\left(K_{t}, \prec^{\prime}\right)$ is said to be a temporal set on $S_{t}$.

Definition 6 Let $S$ be an augmented dynamic trajectory with appropriate temporal ordering $\prec^{\prime}$. Let $\left(K_{t^{\prime}} \prec^{\prime}\right)$ be a temporal set on $S_{t}$. A discrete Z-number in the universe $K_{t}$ is called a temporal discrete $Z$-number and is denoted as $Z_{t}=\left(A_{t} B_{t}\right)$.

The following Lemma, theorem, and corollary lead to temporal discrete Z-numbers as a class of ordered discrete Z-numbers.

Lemma 1 Let $S_{t}$ be an augmented trajectory, then every temporal ordering $\prec^{\prime}$ on $S_{t}$ is a partial ordering on $S_{t}$.

Proof Let $C$ be an augmented trajectory with the temporal ordering $\prec^{\prime}$. Based on Definition 5 of temporal ordering, the relation $\prec^{\prime}$ on $S_{t} \times S_{t}$ generated by $\prec$ has the relation

$$
\left(Z_{1}, t_{1}\right) \prec^{\prime}\left(Z_{2}, t_{2}\right), \Leftrightarrow t_{1} \prec t_{2}
$$


Now, we want to show that its reflexive, antisymmetry and transitive for any $\left(Z_{1}, t_{1}\right),\left(Z_{2}, t_{2}\right),\left(Z_{3}, t_{3}\right) \in S_{t}$ for $t_{1}$, $t_{2} t_{3} \in t$.

1. Reflexive: $\left(Z_{1}, t_{1}\right) \prec^{\prime}\left(Z_{1}, t_{1}\right)$ is true since $(T, \prec)$ is linearly ordered.

2. Antisymmetry: Suppose $\left(Z_{1}, t_{1}\right) \prec^{\prime}\left(Z_{2}, t_{2}\right)$ and $\left(Z_{2}, t_{2}\right) \prec^{\prime}\left(Z_{1}, t_{1}\right)$, this impliest $t_{1} \prec t_{2}$ and $t_{2}$ $\prec t_{1} \Rightarrow t_{1}=t_{2}$ since $(T, \prec)$ is linearly ordered. Therefore, $\left(Z_{1}, t_{1}\right)=\left(Z_{2}, t_{2}\right)$.

3. Transitive: Suppose $\left(Z_{1}, t_{1}\right) \prec^{\prime}\left(Z_{2}, t_{2}\right)$ and $\left(Z_{2}, t_{2}\right) \prec^{\prime}\left(Z_{3}, t_{3}\right)$, this implies $t_{1} \prec t_{2}$ and $t_{2} \prec t_{3} \Rightarrow t_{1} \prec t_{3}$ since $R_{t} \subseteq S_{t}$ is linearly ordered. Therefore, $\left(Z_{1}, t_{1}\right) \prec^{\prime}\left(Z_{3}, t_{3}\right)$.

Hence the temporal ordering $\prec^{\prime}$ on $S_{t}$ is a partial ordering on $S_{t}$.

Theorem 1 Let $S$ be an augmented trajectory, then every temporal ordering $\prec^{\prime}$ on $S_{t}$ is linearly ordering on $S_{t}$.

Proof By lemma 1, the temporal ordering $\prec^{\prime}$ on $S_{t}$ is a partial ordering on $S_{t}$. For any distinct elements of $S_{t}$ i.e. $\left(Z_{1}, t_{1}\right) \neq\left(Z_{2}, t_{2}\right)$, then there exist $g\left(t_{1}\right)=Z_{1}$ and $g\left(t_{2}\right)=$ $Z_{2}$ when $t_{1} \neq t_{2}$. Since $(T, \prec)$ is linearly ordered, then $t_{1} \prec$ $t_{2}$ or $t_{2} \prec t_{1}$. This implies that $\left(Z_{1}, t_{1}\right)$ must precedes $\left(Z_{2}\right.$, $\left.t_{2}\right)$ i.e. $\left(Z_{1}, t_{1}\right) \prec^{\prime}\left(Z_{2}, t_{2}\right)$ or $\left(Z_{2}, t_{2}\right) \prec^{\prime}\left(Z_{1}, t_{1}\right)$. Hence, the temporal ordering $\prec^{\prime}$ on $S_{t}$ is a linearly ordering on $S_{t}$.

Corollary 1 Every temporal discrete Z-number is an ordered discrete Z-number.

Proof By Lemma 1 and Theorem 1 the pair $(S$, $\prec^{\prime}$ is linearly ordered. Furthermore, by Definition 5 of temporal ordering $\prec^{\prime}$ is defined as $\left(Z_{1}, t_{1}\right) \prec^{\prime}\left(Z_{2}\right.$, $\left.t_{2}\right) \Leftrightarrow t_{1} \prec t_{2} \forall\left(Z_{1}, t_{1}\right),\left(Z_{2}, t_{2}\right) \in S_{t}$ where $Z_{1}, Z_{2}$ are ordered discrete Z-numbers. For any $R_{t} \subseteq S_{t}$ where $\left(R_{t}, \prec\right)$ is a temporal set on $S_{t}$, which is linearly ordered. By Definition 6, a discrete Z-number say $Z \in R_{t}$ is called a temporal discrete $Z$-number. Therefore, this means that it must be an ordered discrete Z-number by Definition 5 . Hence, we can simply say that by Lemma 1 , Theorem 1 , Definition 5 and 6, every temporal discrete Z-number is an ordered discrete $\mathrm{Z}$-number.

Therefore, the time variable $t$ in the temporal discrete Z-number is tracking the changes that occurred in the dynamic trajectory of the motion of an EEG signal at different time instant or intervals. These changes are represented by the membership function of the components of temporal discrete Z-number. Moreover, the time variable is not active in any calculation process, that is, is only serving as a representation variable.
METHOD OF MEASURING UNCERTAINTY FOR TEMPORAL DISCRETE Z-NUMBER

The method of measuring uncertainty for temporal discrete Z-number with respect to the time of occurrence is proposed by utilizing the method of measuring the uncertainty for Z-number presented in Kang et al. (2018). Temporal discrete Z-number is a discrete Z-number created from the universe whose ordered in time, therefore, the measure of uncertainty for TDZ will also serve as the measure of uncertainty about the time of occurrence of that particular TDZ.

The method of measuring uncertainty for linguistic temporal discrete Z-number with respect to the time of occurrence is defined as:

Definition 7 Let $Z^{t_{i}}$ be a temporal discrete Z-number, then the uncertainty measure for $Z^{t_{i}}$ is defined as:

$$
H\left(Z^{t_{i}}\right)=\left[H\left(A^{t_{i}}\right)+\frac{1}{2}\left(H\left(B^{t_{i}}\right)+1-x_{B^{t_{i}}}^{*}\right)\right]
$$

where $0 \leq i \leq n, H\left(A^{t i}\right)$ and $H\left(B^{t i}\right)$ are the restriction and reliability given as

$$
H\left(A^{t_{i}}\right)=\frac{N\left(A^{t_{i \cap} A^{t_{i}}{ }^{c}}\right)+\left|X_{A_{i}}\right|-1}{N\left(A^{t_{i \cup A}{ }^{t^{c}}}\right)+\left|X_{A} \mathrm{t}_{i}\right|-1}
$$

such that $N\left(A^{t_{i}} \cap A^{t_{i}{ }^{c}}\right)=\min \left\{A^{t_{i}}, A^{t_{i}{ }^{c}}\right\}$, and $N\left(A^{t_{i}} \cup A^{t_{i}{ }^{c}}\right)$ $=\max \left\{A^{t_{i}}, A^{t_{i}{ }^{c}}\right\}$ and $\left.A^{t_{i}{ }^{c}}\right\}$ the complementary set of $A^{t_{i}}$. The $N(\cdot)$ is the sum and $X_{A^{t_{i}}}$ is the cardinality of the discrete fuzzy number. The $H\left(B^{t_{i}}\right)$ is defined in a similar manner.

The term $1-x_{B}^{*} t_{i}$ in (6) is the inherent uncertainty, where $x_{B}^{*} t_{i}=\left\{x \in X_{B_{i}} \mid \mu_{B^{t_{i}}}=\operatorname{hgt}\left(B^{t_{i}}\right)\right\}$ is the defuzzification of the last maxima. The $\left.\operatorname{hgt}\left(B^{t_{i}}\right)\right\}$ is the height of the discrete fuzzy number $B^{t_{i}}$.

\section{IMPLEMENTATION}

The implementation process of the proposed concept, i.e. temporal discrete Z-number to analyze EEG signal data of an epileptic seizure involves two stages. However, firstly there is a need to establish a relationship between temporal Z-number and type-2 temporal fuzzy set. Then, we can apply a Z-number clustering algorithm, which is introduced in Aliev and Guirimov (2018) to estimate the membership function of temporal discrete Z-number. The final stage is to calculate the measure of uncertainty about the time of occurrence with respect to the membership function of the obtained temporal discrete Z-numbers.

The following definitions are motivated by the definitions of ordered fuzzy and temporal fuzzy sets outlined in Kosanovic (1996). 
Definition 8 Let $E$ be a type-2 fuzzy set in the universe $P$ with a corresponding membership function $\mu_{E}: P \rightarrow[0,1]$. A pair $(E, \prec)$ is called an ordered type-2 fuzzy set if there exists a relation $\prec \operatorname{such}$ that $(P, \prec)$ is a totally ordered set.

Basically, a temporal type-2 fuzzy set is a type-2 fuzzy set generated from a universal set whose elements are ordered in time.

Definition 9 Let $S_{t}$ be an augmented dynamic trajectory with appropriate temporal ordering $\prec^{\prime}$. Let $\left(K_{t}, \prec^{\prime}\right)$ be a temporal set on $S_{t}$. A type-2 fuzzy set in the universe $K_{t}$ is called a type- 2 temporal fuzzy set which is denoted as $E_{t}$.

Temporal discrete Z-numbers can be represented by type- 2 temporal fuzzy set by using and modifying the method of representing Z-number by a type-2 fuzzy set as in Aliev and Kreinovich (2017). Basically, arithmetic operations on Z-number (discrete or continuous) are the same for temporal Z-number (discrete or continuous), since temporal Z-numbers are Z-numbers ordered with time. A similar process is adopted for type- 2 and type2 temporal fuzzy sets. Consequently, Zadeh's extension principle can be applied to temporal discrete Z-numbers as well.

\section{APPLY Z-NUMBER CLUSTERING ALGORITHM}

In most applications, the feature space trajectory is given by a set of samples or feature vectors uniformly distributed in time. Then, the estimation for membership function can be addressed as a pattern recognition problem.
The region of attraction is obtained via partitioning the samples of the feature space trajectory into classes. Since the membership function of temporal discrete Z-number consists of fuzziness and probability, the conventional soft clustering methods such as fuzzy C-means cannot be considered. Hence, the Z-number clustering algorithm based on type-2 fuzzy set in Aliev and Guirimov (2018) is used for the purpose.

\section{MEASURE THE UNCERTAINTY FOR TIME OF OCCURRENCE}

By using the estimated membership functions of the components of temporal discrete Z-number, the measure of uncertainty for the time of occurrence can be determined using (6). The method is developed for measuring the uncertainty for Z-numbers (discrete or continuous) in the first place. Since temporal discrete $Z$-number is a discrete Z-number created from a universe whose elements are ordered with time, therefore, the measure of uncertainty for temporal discrete Z-number is also served as the measure of uncertainty for the time of occurrence for a given temporal discrete $\mathrm{Z}$-number.

\section{NUMERICAL EXAMPLE}

Some of the data used in this work are obtained from Aliev and Guirimov (2018) to illustrate the procedure for analyzing the EEG signal of an epileptic seizure. The data is listed in Table 1. By applying the mentioned Z-number clustering algorithm, one is able to partition the data into clusters. They are represented by the membership function of temporal discrete Z-number.

TABLE 1. Fragment of EEG signal data set of seizure

\begin{tabular}{ll}
\hline$x_{i}, 1$ & $x_{i}, 1$ \\
$\ldots$ & $\ldots$ \\
1.05 & 0.774906 \\
1.10 & 0.822311 \\
1.15 & 0.87494 \\
1.20 & 0.933029 \\
1.25 & 0.996711 \\
1.30 & 1.066098 \\
1.35 & 1.141221 \\
1.40 & 1.22203 \\
1.45 & 1.308371 \\
1.50 & 1.399982 \\
1.55 & 1.496474 \\
$\ldots$ & $\ldots$ \\
4 & 7.386384 \\
\hline
\end{tabular}


Firstly, in order to obtain a type-2 temporal fuzzy set cluster, fuzzy fuzzifier is used as shown in Figure 1.

A type- 2 membership function of one of the clusters (cluster 2) is described in Figure 2.

The membership function of the first component of temporal discrete Z-number; i.e. $A^{t}$ is obtained as a centroid of type-2 data-to-cluster membership function as shown in Figure 3.

The second component of temporal discrete Z-number, i.e. $B^{t}$ is determined by constructing a probability density function using the obtained membership function of $A^{t}$. Figure 4 demonstrates the probability density function.

Lastly, by computing the probability measure for $A^{t}$, the membership function of $B^{t}$ is constructed and demonstrated in Figure 5.

Supposed the membership values for $A^{t}$ and $B^{t}$ with respect to dimension are represented as follows,

$$
A^{t}=0 / 5+0.75 / 6+1 / 7+0.75 / 8+0 / 9
$$

and

$$
=0 / 0.1+0.5 / 0.2+0.75 / 0.3+1 / 0.4+0 / 0.5
$$

The membership values are then used to determine the measure of uncertainty for $Z^{t}$ in dimension with respect to the time of occurrence using (6). Firstly, both $H\left(A^{t_{i}}\right)$ and $H\left(B^{t_{i}}\right)$ are calculated using (7);

$$
\begin{gathered}
H\left(A^{t_{i}}\right)=\frac{N\left(A^{t_{i}} \cap A^{t_{i}}{ }^{c}\right)+\left|X_{A^{t_{i}}}\right|-1}{N\left(A^{t_{i}} \cup A^{t_{i}}\right)+\left|X_{A^{t_{i}}}\right|-1} \\
=\frac{0+0.25+0+0.25+0+5-1}{1+0.75+1+0.75+1+5-1} \\
=0.5294
\end{gathered}
$$

and

$$
\begin{gathered}
H\left(B^{t_{i}}\right)=\frac{N\left(B^{t_{i}} \cap B^{t_{i}}{ }^{c}\right)+\left|X_{B^{t_{i}}}\right|-1}{N\left(B^{t_{i}} \cup B^{t_{i}^{c}}\right)+\left|X_{B^{t_{i}}}\right|-1} \\
=\frac{0+0.5+0.25+0+0+5-1}{1+0.5+0.75+1+1+5-1} \\
=0.5586
\end{gathered}
$$

Then, by substituting $H\left(A^{t_{i}}\right)$ and $H\left(B^{t_{i}}\right)$ into (6), the uncertainty measure of temporal discrete Z-number is obtained;

$$
\begin{gathered}
H\left(Z^{t_{i}}\right)=\frac{1}{2}\left[H\left(A^{t_{i}}\right)+\frac{1}{2}\left(H\left(B^{t_{i}}\right)+1-x_{B}^{*} t_{i}\right)\right] \\
=\frac{1}{2}\left[0.5294+\frac{1}{2}(0.5757+1-0.4)\right] \\
=0.5586
\end{gathered}
$$

Analogously, the measure of uncertainty for $Z^{t_{i}}$ in $y$ dimension with respect to the time of occurrence is determined in the same manner earlier.

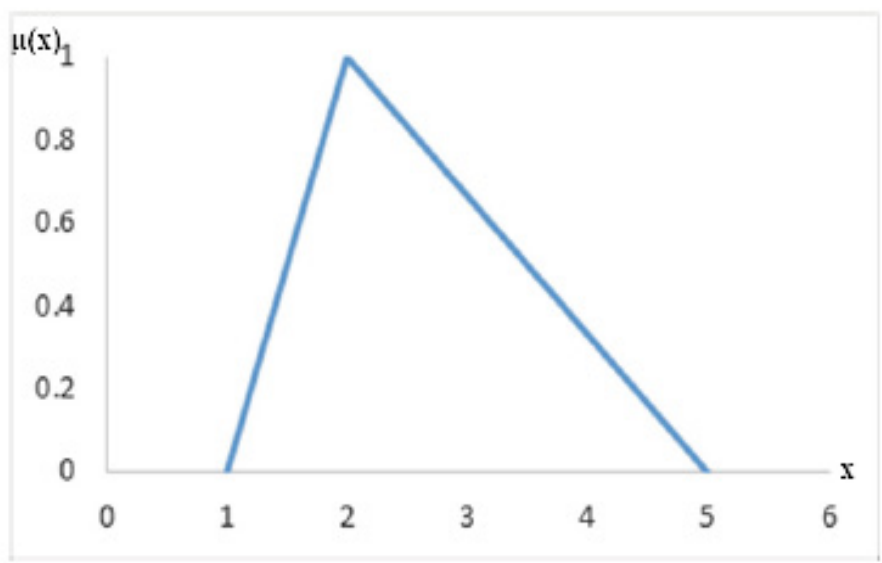

FIGURE 1. Fuzzy fuzzifier membership function 

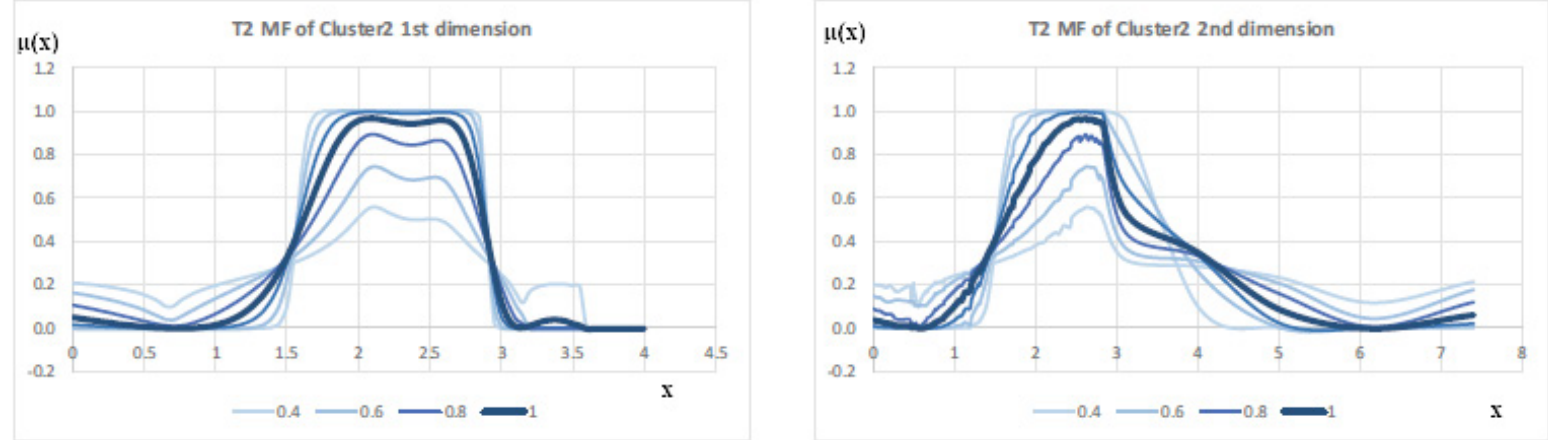

FIGURE 2. Type-2 data-to-cluster membership function for $x$ and $y$ dimension of cluster 2
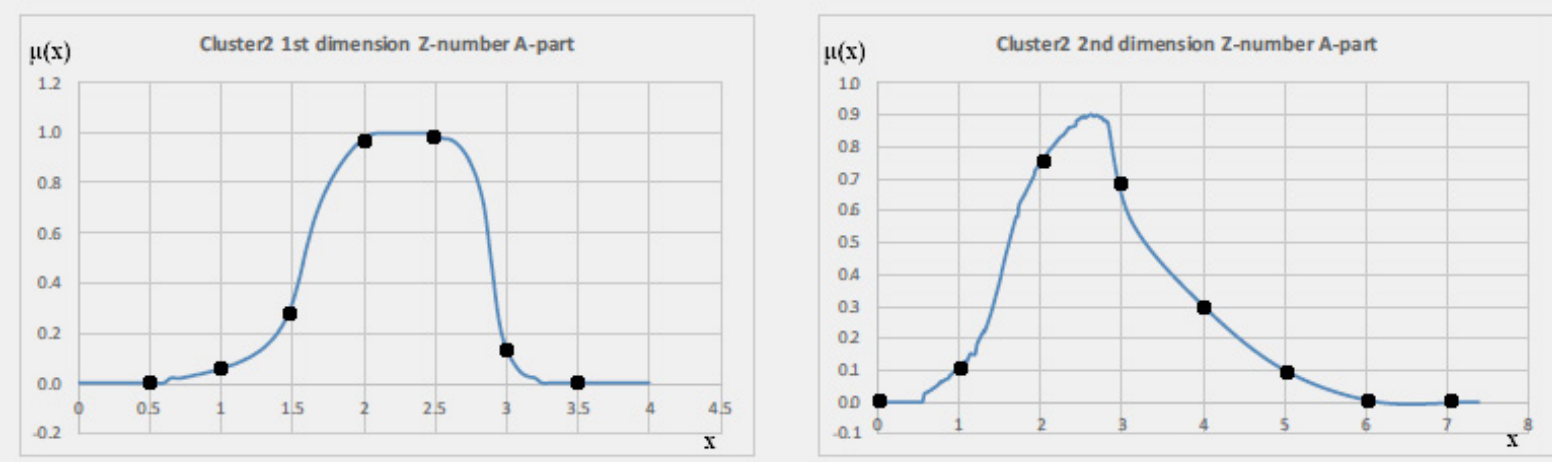

FIGURE 3. Membership function of $A^{t}$ for $x$ and $y$ dimension
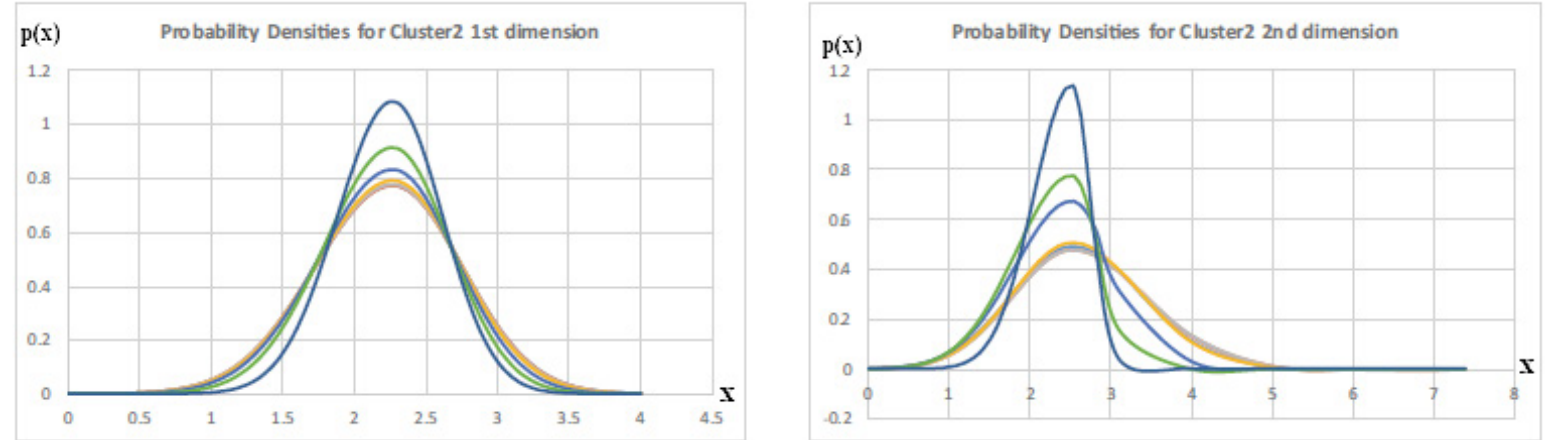

FIGURE 4. Probability density function for $x$ and $y$ dimension 


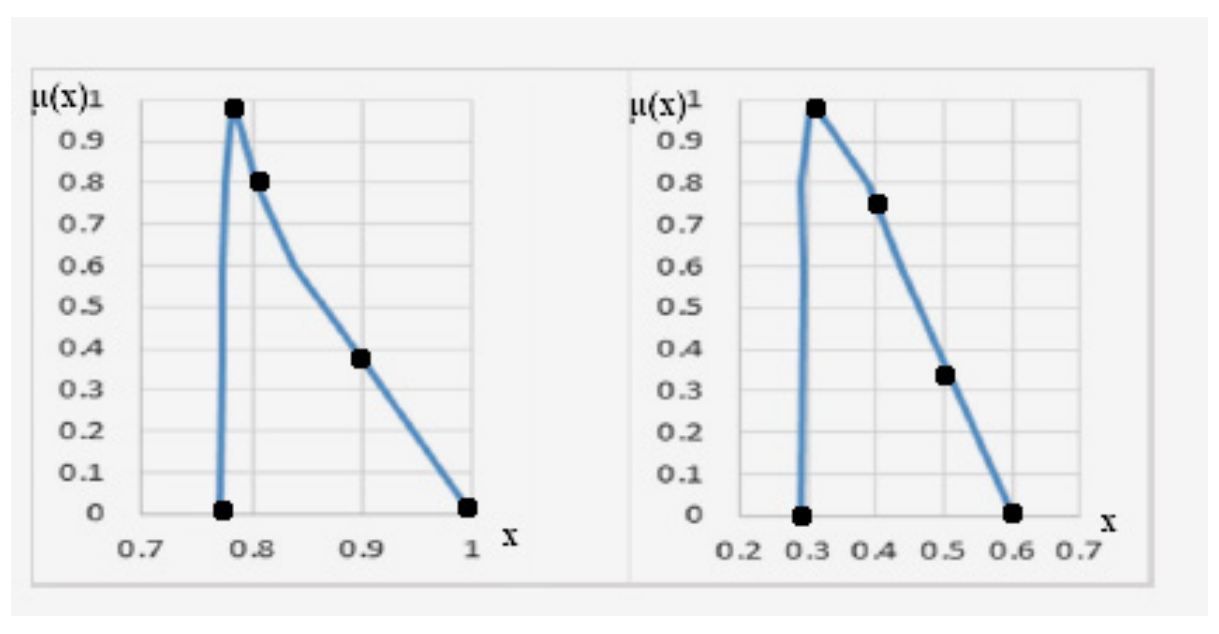

FIGURE 5. Membership function of $B^{t}$ for $x$ and $y$ dimension

\section{CONCLUSION}

The proposed concept is introduced to serve as a tool for modeling dynamic motions of complex physical systems particularly EEG signal data of epileptic seizures. It has the ability to measure the uncertainty with respect to the time of occurrence. Finally, a numerical example is presented to illustrate the procedure and feasibility of the proposed concept.

\section{ACKNOWLEDGEMENTS}

This work has been supported by UTM COE GRANT, 04G05 and the first author is financially supported by the Ministry of Education of Nigeria.

\section{REFERENCES}

Abdullahi, M., Ahmad, T. \& Ramachandran, V. 2020. Ordered discrete and continuous Z-numbers. Malaysia Journal of Fundamental and Applied Sciences (In Press).

Aliev, R. \& Guirimov, B. 2018. Z-number clustering based on general type-2 fuzzy sets. In International Conference on Theory and Applications of Fuzzy Systems and Soft Computing. Springer, Cham. pp. 270-278.

Aliev, R.A. \& Kreinovich, V. 2017. Z-Numbers and type-2 fuzzy sets: A representation result. Intelligent Automation \& Soft Computing 24(1): 1-5.

Aliev, R.A., Alizadeh, A.V. \& Huseynov, O.H. 2015. The arithmetic of discrete Z-numbers. Information Sciences 290: 134-155.

Casasnovas, J. \& Riera, J.V. 2006. On the addition of discrete fuzzy numbers. In Proceedings of the 5th WSEAS International Conference on Telecommunications and Informatics. World Scientific and Engineering Academy and Society (WSEAS). pp. 432-437.
Fauziah, B.Z. 2008. Dynamic profiling of EEG data during seizure using fuzzy information space. Universiti Teknologi Malaysia, Ph.D. Thesis (Unpublished).

Kang, B., Deng, Y., Hewage, K. \& Sadiq, R. 2018. A method of measuring uncertainty for Z-number. IEEE Transactions on Fuzzy Systems 27(4): 731-738.

Kosanovic, B.R., Chaparro, L.F. \& Sclabassi, R.J. 1996. Signal analysis in fuzzy information space. Fuzzy Sets and Systems 77(1): 49-62.

Kosko, B. 1990. Fuzziness vs. probability. International Journal of General System 17(2-3): 211-240.

Nagypál, G. \& Motik, B. 2003. A fuzzy model for representing uncertain, subjective, and vague temporal knowledge in ontologies. In OTM Confederated International Conferences on the Move to Meaningful Internet Systems. Springer, Berlin, Heidelberg. pp. 906-923.

Mendel, J.M. \& John, R.B. 2002. Type-2 fuzzy sets made simple. IEEE Transactions on Fuzzy Systems 10(2): 117127.

Rangasamy, P. 2009. A note on properties of temporal intuitionistic fuzzy sets. In Notes on IFS Conference Proceedings. 15(1): 42-48.

Sharmila, A. \& Geethanjali, P. 2019. A review on the pattern detection methods for epilepsy seizure detection from EEG signals. Biomedical Engineering/Biomedizinische Technik 64(5): 507-517.

Zadeh, L.A. 2011. A note on Z-numbers. Information Sciences 181(14): 2923-2932.

Mujahid Abdullahi \& Tahir Ahmad*

Department of Mathematical Sciences, Faculty of Science

Universiti Teknologi Malaysia

81310 UTM Skudai, Johor Darul Takzim

Malaysia 
Mujahid Abdullahi

Department of Mathematics and Computer Science Faculty of Natural and Applied Sciences

Sule Lamido University 048 SLU Kafin Hausa, Jigawa Nigeria

Vinod Ramachandran

Level 23, Menara CIMB, Jalan Stesen Sentral 2

Kuala Lumpur Sentral

50470 Kuala Lumpur, Federal Territory

Malaysia
*Corresponding author; email: tahir@ibnusina.utm.my

Received: 15 January 2020

Accepted: 10 May 2020 